\title{
CRECIMIENTO Y DESARROLLO DEL FRUTO DE CHAMPA (Campomanesia lineatifolia Ruiz \& Pavón)
}

\section{GROWTH AND DEVELOPMENT OF CHAMPA FRUIT (Campomanesia lineatifolia Ruiz \& Pavón)}

\author{
Helber Enrique Balaguera1, Javier Giovanni Álvarez², Diana Carolina Bonilla ${ }^{3}$
}

\begin{abstract}
${ }^{1}$ I.A. Grupo de Investigaciones Agrícolas, Facultad de Ciencias Agropecuarias, Universidad Pedagógica y Tecnológica de Colombia. Av. Central del Norte km 0 vía Paipa, Tunja, Colombia. e-mail: enrique_balaguera@yahoo.com, ${ }^{2}$ Profesor asistente. Grupo de Investigaciones Agrícolas, Facultad de Ciencias Agropecuarias, Universidad Pedagógica y Tecnológica de Colombia. Av. Central del Norte km 0 vía Paipa, Tunja, Colombia. e-mail: jgalvarezh@gmail.com, ${ }^{3}$ I.A. Grupo de Investigaciones Agrícolas, Facultad de Ciencias Agropecuarias, Universidad Pedagógica y Tecnológica de Colombia. Av. Central del Norte Km 0 vía Paipa, Tunja, Colombia. e-mail: dicabo83@hotmail.com
\end{abstract}

Rev. U.D.C.A Act. E Div. Cient. 12 (2): 113-123, 2009

\section{RESUMEN}

La champa es un árbol frutal perteneciente a la familia myrtaceae. Su fruto es una baya pequeña altamente perecedera, de agradable saber dulce acidulado, bastante aceptada por los consumidores. No obstante, las investigaciones sobre esta fruta son muy pocas y no se ha determinado el comportamiento fisicoquímico durante el crecimiento y el desarrollo del fruto, que permita establecer un manejo técnico adecuado y, de esta manera, evitar pérdida de frutos. Se seleccionaron frutos del tercio medio de árboles adultos de champa y, cada 15 días, desde la formación del fruto, se realizaron mediciones de parámetros físicos y químicos. Se determinó que desde la floración hasta la cosecha, el fruto tarda 160 días. Además, la acumulación de masa fresca y seca siguió una curva sigmoidal simple. Una vez que el fruto creció y se desarrolló, aumentó la masa fresca y seca, los sólidos solubles totales (SST), el pH y la relación de madurez (RM), mientras que la acidez total titulable (ATT) y la firmeza disminuyeron. El fruto es bastante ácido, pero tiene un alto contenido de SST, que hace que tenga un uso potencial en la elaboración de jugos industriales y que su aceptación por el consumidor sea favorable. La firmeza del fruto al momento de la cosecha es muy baja, lo cual, limita el mercadeo en fresco y la vida poscosecha de la champa.

Palabras clave: Cultivo perenne, Myrtaceae, frutas tropicales, perecedero, jugo.

\section{SUMMARY}

The champa is a fruit tree, which belongs to the family Myrtaceae. Its fruit is a small berry, highly perishable of delicious flavour, well accepted by consumers. However, research regarding this fruit is hardly any and, besides, the physicochemical behaviour during fruit growth and development, to establish the adequate management to avoid fruit loss, has not been determined. Fruits of the middle third part of champa trees were selected and every 15 days, from its formation on, physical and chemical parameters of the fruits were measured. It was determined that from flowering to harvest the fruit takes 160 days. Furthermore, the accumulation of fresh and dry mass followed a simple sigmoidal curve. Once the fruit grew and developed, the fresh and dry mass, the total soluble solids (TSS), $\mathrm{pH}$ and the maturity ratio (MR) increased, while the total titratable acidity (TTA) and the firmness decreased. The fruit is quite acid, but has a high 
TSS content, which makes that it has a potential use in industrial fruit juice processing and its acceptance by the consumer can be positive. The fruit firmness at harvest is very low, limiting the market for fresh and post-harvest life of champa fruit.

Key words: Perennial crop, Myrtaceae, tropical fruits, perishable, juice.

\section{INTRODUCCIÓN}

La champa es un árbol frutal (Figura 1.1) poco conocido, perteneciente a la familia Myrtaceae. Es nativo de la Amazonía brasileña, peruana, colombiana y boliviana. Se conoce con diferentes nombres comunes, como michinche, palillo, guayabo Anselmo, guayaba de leche, guayaba de mono y guabiroba o gabiroba (López \& Rodríguez, 1995; Villachica, 1996). El fruto de champa (Figura 1.6) es de sabor y aroma agradable, con abundante pulpa jugosa, dulce acidulada muy apetecida por el consumidor (Villachica, 1996). Crece en condiciones de temperaturas de 22 a $30^{\circ} \mathrm{C}$, con precipitaciones superiores a $1.500 \mathrm{~mm}$ anuales, necesita suelos de texturas francas a arcillosas (Villachica, 1996). En promedio, el árbol empieza a producir a los cinco años. El inicio de la floración (Figura 1.2), en Miraflores (Boyacá), habitualmente ocurre entre marzo y mayo, y la máxima producción se presenta en septiembre (López E Rodríguez, 1995) (Figuras 1.2, 1.3, 1.4, 1.5 y 1.6). La producción anual por árbol va desde los 5kg hasta los 500kg; la cosecha, se hace cada tercer día y se pueden llegar a recolectar hasta $75 \mathrm{~kg}$ por jornada; un árbol puede durar en cosecha hasta 15 días (López E Rodríguez, 1995).

Tradicionalmente, los frutos son recolectados del suelo, ya que a medida que se acercan al punto de maduración se desprenden del árbol (Villachica, 1996). Esto hace que los frutos pierdan su calidad rápidamente y que su comercialización se vea limitada. Además, no se ha determinado el punto óptimo de cosecha, por desconocimiento de los cambios fisicoquímicos del fruto durante su crecimiento y desarrollo, así como la duración de éstos. Por lo cual, algunos frutos se recolectan en diferentes estados de madurez y muchos son desechados porque no cumplen con las características organolépticas exigidas por el consumidor o comercializador, lo que le genera pérdidas económicas al productor. No existen estudios sobre el comportamiento fisicoquímico de los frutos, sin embargo, se asume que tengan un comportamiento similar al de otras mirtáceas, como el araza y la guayaba. Laguado et al. (1999) afirman que en guayaba a medida que los frutos crecen, aumenta el $\mathrm{pH}$, se incrementa la velocidad de degradación de las sustancias celulósicas pécticas, lo que se traduce en la pérdida de la firmeza del fruto y aumento de los azúcares solubles durante el proceso de maduración. El arazá (Hernández et al. 2007) y la guayaba siguen una curva sigmoide simple con tres fases de crecimiento: una inicial de división celular, una fase de crecimiento exponencial de elongación celular y, finalmente, la de maduración (Mercado-Silva et al. 1998; Nakasone $\mathcal{E}$ Paull, 1999).

Por lo anterior, el objetivo de este estudio fue determinar los cambios fisicoquímicos durante el crecimiento y el desarrollo del fruto de champa en el municipio de Miraflores (Boyacá). De esta manera, se logrará planear e implementar recolecciones oportunas, que mantengan las características de calidad propias del fruto, con el fin de dar una eficiente utilización a las más de 370 t que se producen anualmente en la región de Lengupá Boyacá.

\section{MATERIALES Y MÉTODOS}

La investigación en la fase de campo, se realizó durante el 2008, en la finca "La Playa", de la vereda Ayatá, en el municipio de Miraflores, Boyacá, situado a 5¹1' de latitud norte y a $73^{\circ} 08^{\prime}$ de longitud oeste, a una altura de $1432 \mathrm{msnm}$ con una temperatura promedio de $20^{\circ} \mathrm{C}$ y una precipitación que oscila entre 2000 y 3000mm, ocurrida en un periodo monomodal, comprendido entre abril y septiembre.

Los frutos se recolectaron de árboles, entre los cinco y diez años de edad, sembrados sin manejo tecnificado, como sombrío para el cultivo de café, a distancias aproximadas de $8 \times 8 \mathrm{~m}$. Durante el crecimiento y el desarrollo del fruto, se hicieron ocho muestreos cada quince días, empezando desde los 55 días después de la floración (DDF), debido a que antes de este tiempo el tamaño de los frutos no permitió realizar ningún tipo de medición; los frutos presentaron un diámetro polar y ecuatorial de $1,51 \pm 0,2 \mathrm{~cm}$ y $2,05 \pm 0,3 \mathrm{~cm}$, respectivamente y se llevaron a cabo diez repeticiones por variable. Para todas las determinaciones físicas, se utilizaron 80 frutos, diez por muestreo, un fruto por repetición. Para todas las determinaciones químicas, se 


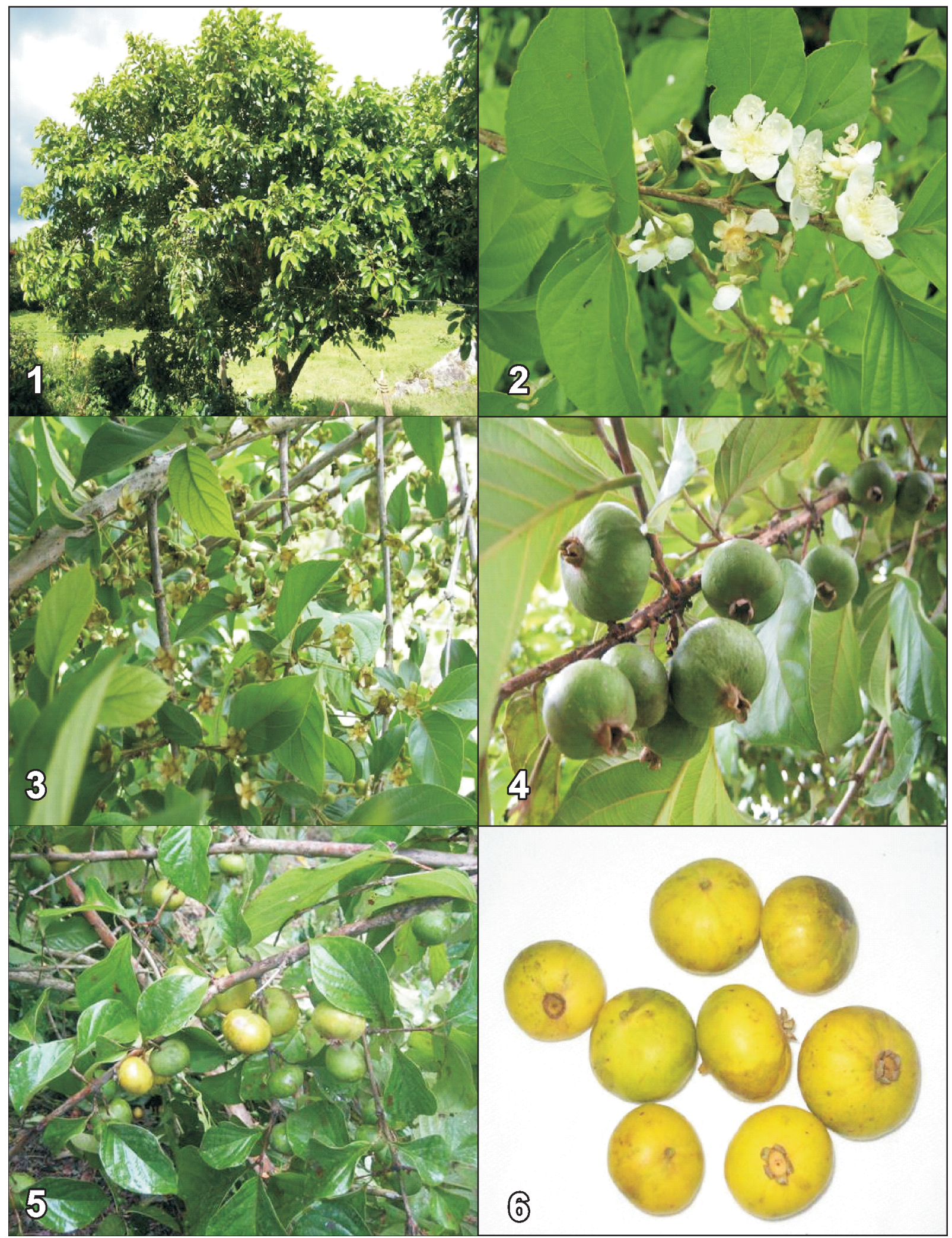

Figura 1. Árbol de champa y diferentes estados de crecimiento del fruto. 1.1. Árbol adulto que inicia producción; 1.2. Floración; 1.3. Formación del fruto; 1.4. Llenado de fruto; 1.5. Frutos en diferentes estados de madurez; 1.6. Fruto con madurez de consumo. 
tomaron 440 frutos, 90 frutos en la primera medición y, en cada época, se disminuyó en diez el número de frutos, debido a que estos a medida que crecieron producían mayor cantidad de jugo, y la extracción del mismo se hacía con mayor facilidad. El total de frutos muestreados fue de 520, tomados de 20 árboles seleccionados al azar. Del tercio medio de cada árbol, se recolectaron 26 frutos, previamente marcados.

Las variables medidas fueron: masa fresca: se determinó en una balanza de precisión, con aproximación de 0,01g; masa seca: masa de los frutos después de someterlos en una mufla a $90^{\circ} \mathrm{C}$ durante 36 horas; firmeza del fruto (Newtons $(\mathrm{N})$ ): mediante la utilización de un penetrómetro digital PCE-PTR200; pH: se midió con un potenciómetro Orión modelo 420; sólidos solubles totales (SST): medición realizada con un refractómetro marca Atago; acidez total titulable (ATT), a través de la titulación con $\mathrm{NaOH} \mathrm{0,1} \mathrm{N} \mathrm{hasta} \mathrm{pH} \mathrm{8,2,} \mathrm{y} \mathrm{se} \mathrm{expresó}$ como ácido málico y, finalmente, la relación de madurez (RM), se estableció como la relación SST/ATT.

La determinación de las variables físicas y químicas a lo largo del desarrollo y la maduración de los frutos, se ejecutaron en los laboratorios de Fisiología Vegetal de la
UPTC, sede Tunja. Para las variables medidas, se efectuó un análisis descriptivo en, el cual, se estipuló el promedio y la desviación estándar con el software SAS v. 8.1e.

\section{RESULTADOS Y DISCUSIÓN}

El comportamiento de la masa seca de los frutos de champa, se ajustó al modelo de crecimiento no lineal tipo logístico, debido a que presentó menor cuadrado medio residual del error comparado con los modelos de Gompertz y Gaussiano. Los frutos mostraron incrementos continuos en la ganancia de masa seca a través del tiempo; sin embargo, desde los 55 DDF hasta los 85 DDF esta ganancia fue baja; de los 145 DDF hasta los 160 la ganancia fue representativa, mientras que de los 85 DDF a los 145 DDF, se acumuló la mayor cantidad de masa, alcanzando el $67,56 \%$ de la masa total. A los $55 \mathrm{DDF}$, los frutos pesaron $0,52 \pm 0,07 \mathrm{~g}$. Al momento de la cosecha los frutos adquirieron una masa de 5,21 $\pm 0,55 \mathrm{~g}$ (Figura 2).

El aumento de la masa seca del fruto de champa puede ser explicado por la formación de proteínas, azúcares y otras sustancias acumuladas durante el desarrollo del

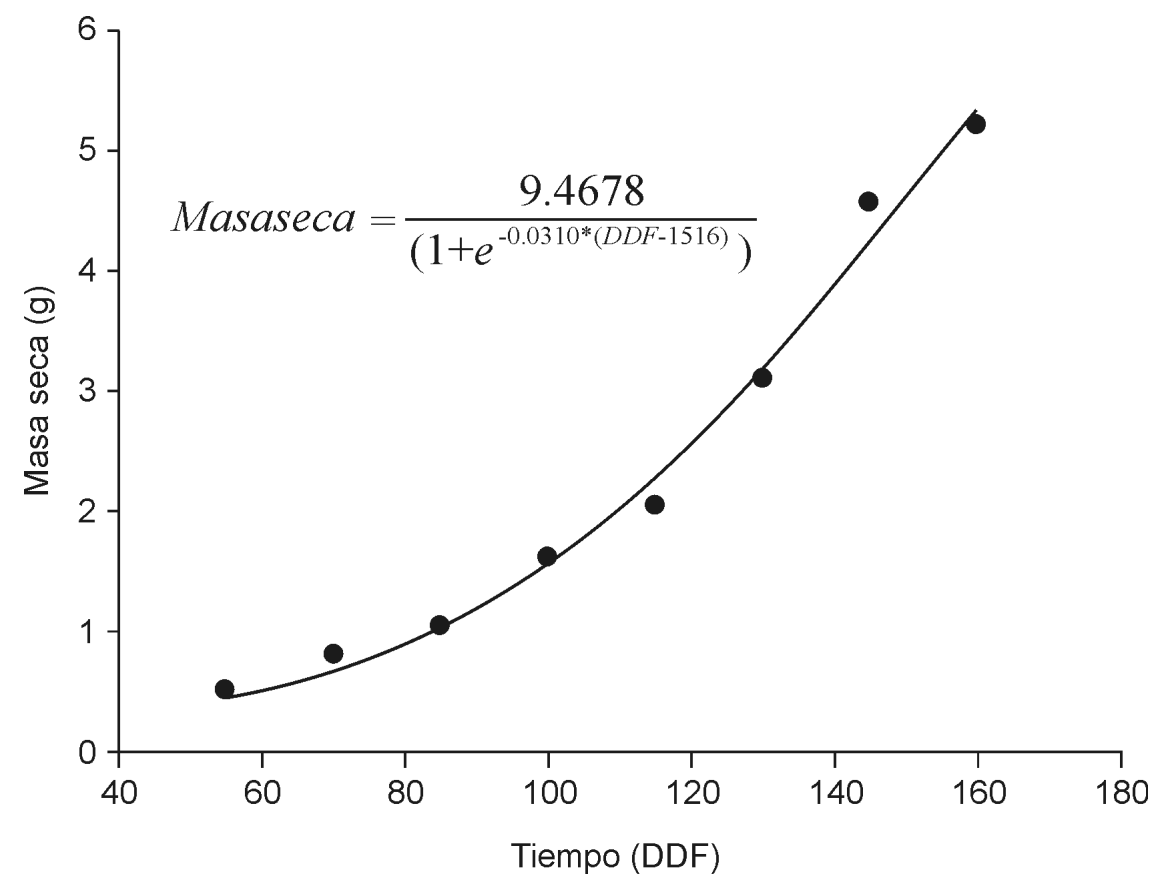

Figura 2. Comportamiento de la masa seca en el crecimiento y desarrollo de frutos de champa. 
fruto, tal como lo afirma Días (2001). Se ha determinado que durante la primera fase del crecimiento de los frutos, el contenido de clorofila total aumenta, con lo cual, el fruto muestra una coloración verde, ocurre un crecimiento acelerado y un predominio de la síntesis de clorofilas sobre la de antocianinas (Hernández-Gil $\mathcal{E}$ Bautista, 1977). Así mismo, la síntesis de hormonas de crecimiento, se encuentra estimulada, lo que provoca una rápida división celular; acorde con el incremento en el contenido de auxinas (Coombe, 1960). Kender et al. (1970) encontraron una adición en el contenido de auxina y de giberelina en la primera fase y un aumento de auxinas en la segunda fase del desarrollo de Vaccinium angustifolium AIT.

La masa fresca registró una cinética similar a la observada en la masa seca, ajustándose a un modelo logístico. Esta curva sigmoidal simple propia de frutos carnosos (Salisbury \& Ross, 1994), se caracterizó porque la acumulación de masa fresca fue exponencial con el tiempo, con una rapidez de crecimiento baja al principio (70 DDF), pero aumentó en forma continua hasta los 85 DDF; luego, siguió una fase lineal, momento en el que, la masa ascendió considerablemente hasta los 145 DDF y, finalmente, se presentó la fase de maduración, que fue hasta los 160 DDF, donde la ganancia de masa fue baja (Figura 3). En esta misma fase, se observó el cambio en la coloración de la corteza, el cual fue pasando gradualmente de verde oscuro a amarillo; cuando el fruto obtiene esta última coloración está listo para ser cosechado y consumido (López E Rodríguez, 1995).

Este proceso, según Medeiros E Raseira (1998) está acompañado de la producción de etileno y otras sustancias volátiles, de cambios en el color, de la permeabilidad de los tejidos y de transformaciones químicas que afectan a los azúcares, los ácidos orgánicos, las proteínas, los compuestos fenólicos, los pigmentos, las pectinas y otras sustancias. Durante la maduración, se obtienen los sabores y los olores específicos, los aumentos en la dulzura y la disminución de la acidez, que se vuelven más pronunciados. También es durante este periodo que se da el ablandamiento de la fruta.

A los 55 DDF, los frutos presentaron una masa fresca de $1,76 \pm 0,45 \mathrm{~g}$, al momento de la cosecha, un valor de $24,8 \pm 1,73 \mathrm{~g}$. Se pudo observar que los frutos de champa mostraron un desarrollo homogéneo con variaciones mínimas en la masa acumulada, por lo cual, el periodo de cosecha se da en un corto tiempo, según

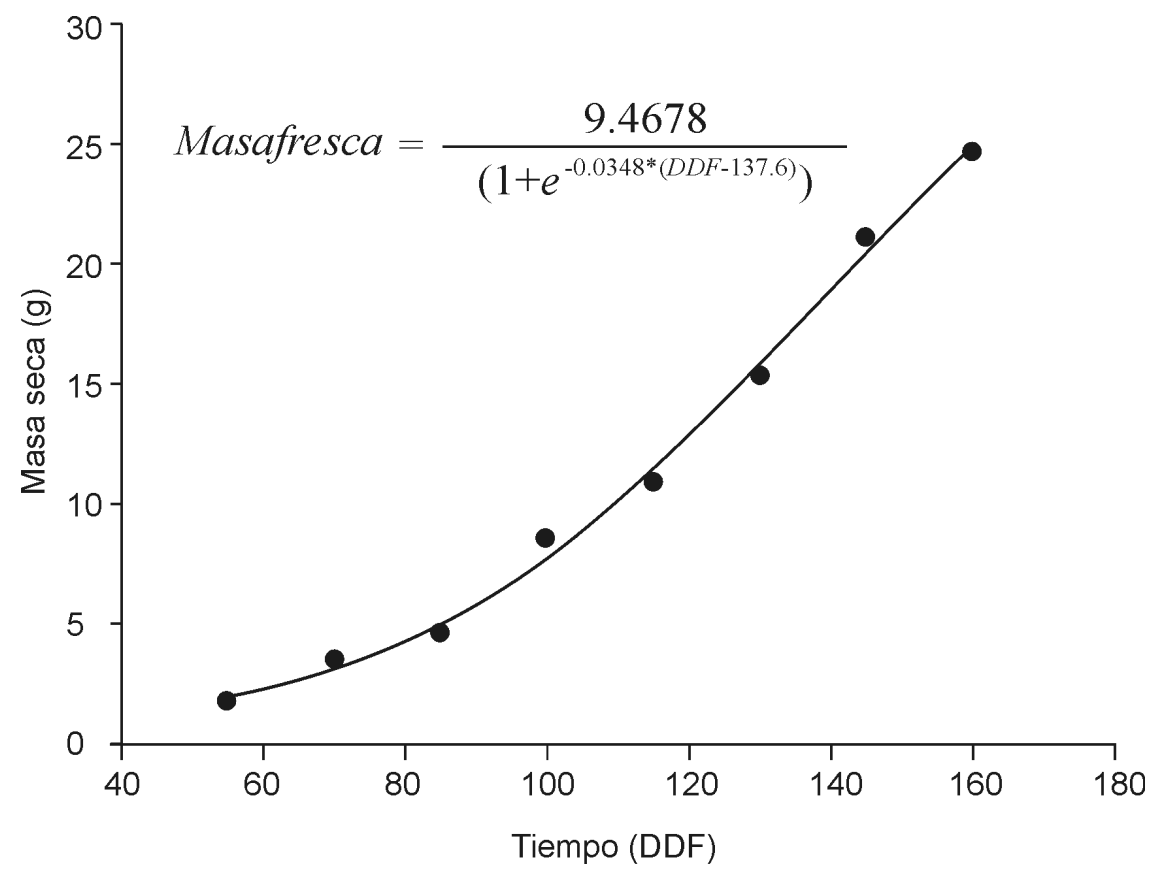

Figura 3. Comportamiento de la masa fresca en el crecimiento y desarrollo de frutos de champa. 
López \& Rodríguez (1995) y el periodo de cosecha de un árbol de champa es de aproximadamente 15 días.

Al inicio del crecimiento del fruto de champa, el aumento de la masa fresca es bajo, debido a que en este periodo ocurre una elevada división celular (Salisbury $E$ Ross, 1994). Luego se genera una elongación celular, influenciada por la plasticidad de las paredes celulares y por la presión de turgencia de las células, responsable del aumento pronunciado en volumen y en masa (Garcés, 1987) y, por último, el crecimiento se detiene durante la fase de maduración. Este comportamiento fue también observado para arazá (Hernández et al. 2007) y guayaba (Nakasone E Paull, 1999).

El contenido de humedad de los frutos de champa es del $79 \%$, inferior que los frutos de Campomanesia phae $(88,8 \%)$ (Vallilo et al. 2005). Esta es una característica común de los frutos de la familia Myrtaceae, los cuales, en su mayoría, son carnosos y suculentos, confirmando lo descrito por Gemtchüjnicov (1976) y Legrand \& Klein (1977), para frutos de la subfamilia Myrtoidea, a la que pertenece la champa. Se evidenció una pérdida de firmeza de forma lineal durante el desarrollo de los frutos, con una disminución de $0,749 \mathrm{~N} \cdot$ día $^{-1}$, manifestándose una alta firmeza en los primeros estados y, luego, una pérdida drástica (Figura 4), pasando de 82,46 $\pm 2,93 \mathrm{~N}$ (55 DDF) a 6,6 \pm 1,08N (160 DDF).
Los frutos exhibieron un ablandamiento durante la maduración, proceso que ocurre en la mayoría de los frutos (Reid, 2002). En las primeras etapas de desarrollo, su firmeza y su consistencia son óptimas; durante la maduración la sustancia adherente de las células, la propectina va degradándose junto con las sustancias pécticas, lo cual, altera la textura y la consistencia del fruto (Osterloh et al. 1996).

En la maduración de los frutos de champa es posible que se expresen muchas enzimas relacionadas con la pared celular que modifican la plasticidad (Öpik E Rolfe, 2005). Las principales enzimas involucradas en este proceso son la poligalacturonasa, celulasa y pectinmetilesterasa (Zarra E Revilla, 1993) y, en algunos casos, glicosidasas (Minic E Jouanin, 2006). Osterloh et al. (1996) atribuyen una disminución elevada de la firmeza del fruto a un metabolismo alto y a una maduración acelerada. Según los mismos autores, este comportamiento se presenta, especialmente, cuando la acumulación del calcio en el fruto es insuficiente y los frutos son muy grandes, lo cual pudo ocasionar también la disminución de la firmeza. La importancia de la firmeza radica en que es uno de los parámetros más importantes para determinar tanto la calidad como el posible manejo y empaque del producto (Kays, 2004), dado que los frutos de champa pierden esta propiedad rápidamente, lo que hace más complicado el manejo.

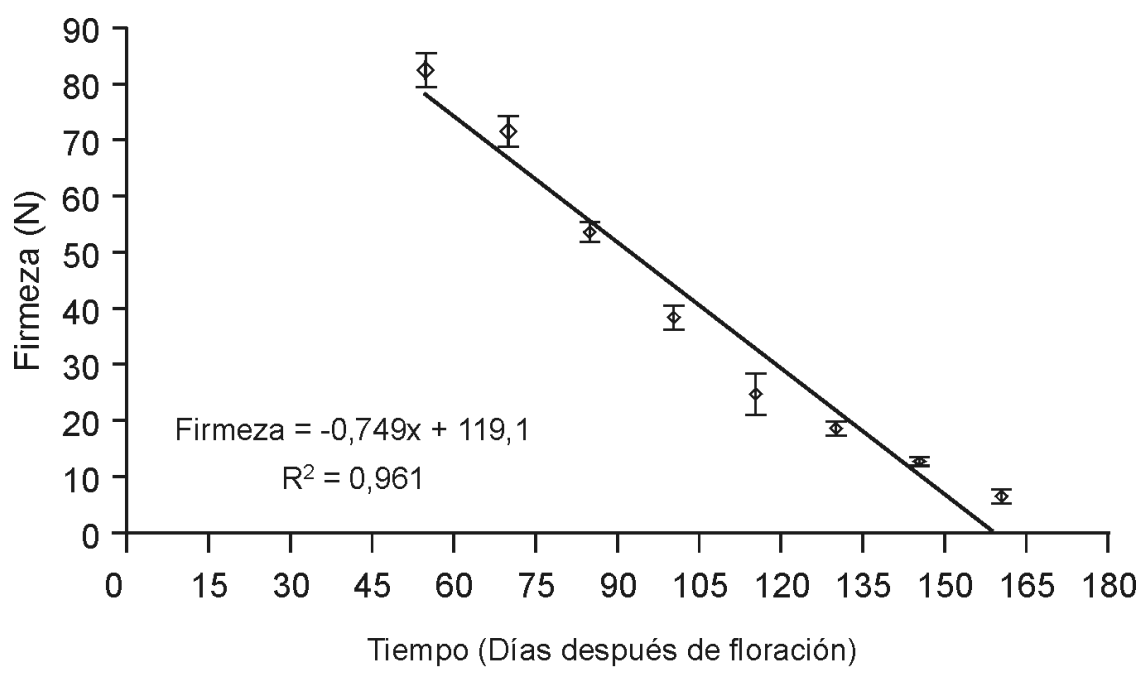

Figura 4. Comportamiento de la firmeza en el crecimiento y desarrollo de frutos de champa. Las barras verticales indican la desviación estándar $(n=10)$. 
El comportamiento del $\mathrm{pH}$ descrito por un polinomio de segundo grado mostró un descenso de los 55 a los 85 DDF y después aumentó hasta los 160 DDF, pasando de 3,72 $\pm 0,19$ a 3,94 $\pm 0,07$ (Figura 5). La disminución del $\mathrm{pH}$ hasta los $85 \mathrm{DDF}$, se debe, posiblemente, a la acumulación de ácidos orgánicos del ciclo de los ácidos tricarboxílicos o a una fijación de $\mathrm{CO}_{2}$ en ácido málico (Vickery, 1952). Por el contrario, el incremento ocurrido en el $\mathrm{pH}$ después de los 85 DDF esta dado, principalmente, por la disminución de los $\mathrm{H}^{+}$a nivel vacuolar, que pasan a conformar esqueletos de azúcares y por el incremento en la actividad de las deshidrogenasas, que favorecen la conversión de ácidos orgánicos a azúcares, utilizados como sustrato para la respiración (Marschner, 2002), lo que trae como consecuencia la disminución de la ATT (Villamizar, 2001).

En los frutos, más del $90 \%$ del volumen celular lo ocupa la vacuola, la cual, usualmente, es muy ácida, con un $\mathrm{pH}$ menor de cinco (Nanos E Kader, 1993), afirmación que coincide con los resultados encontrados en los frutos de champa. El valor de $\mathrm{pH}$ en la cosecha para los frutos de champa es superior a los reportados para C. phae $(\mathrm{pH}=2,91)$ por Vallilo et al. (2005), quienes también afirman que a mayor acidez existe una mayor posibilidad de industrialización de los frutos en forma de dulces.

Del mismo modo, Andrade et al. (1993) mencionan que para la elaboración de néctares, un alto nivel de acidez provoca una elevada dilución del producto y, por consiguiente, un aumento en el rendimiento final de la bebida. Uno de los destinos más comunes de los frutos de champa es la elaboración de jugos caseros (López E Rodríguez, 1995; Villachica, 1996); este uso se debe a su exquisito sabor, mas no a su eficiencia en el rendimiento de la pulpa, pero los resultados ponen de manifiesto que industrialmente la champa también tendría gran aceptación por su alta acidez.

La ATT disminuyó linealmente a una tasa de $0,0312 \% \cdot$ dia $^{-1}$ y pasó de 6,34 \pm 0,22\% (55 DDF) a 3,54 \pm 0,15\% (160 DDF) (Figura 5). Generalmente, los ácidos disminuyen durante la maduración, ya que ellos son sustratos respiratorios o son convertidos a azúcares. De tal forma, pueden ser considerados una fuente de energía y se esperaría que disminuyeran a través de la actividad metabólica, que se desarrolla en la maduración (Heredia et al. 1997). Por el contrario, en Acca sellowiana se encontró un aumento en el contenido de ácidos orgánicos (Rodríguez et al. 2006), concordando con lo reportado en guayaba, en la que la acidez titulable aumenta progresivamente hasta la cosecha (Mercado et al. 1998).

Los SST se incrementaron de forma continua durante el crecimiento, comportamiento que se ajustó a una regresión lineal: al inicio, el valor fue de 7,7 \pm

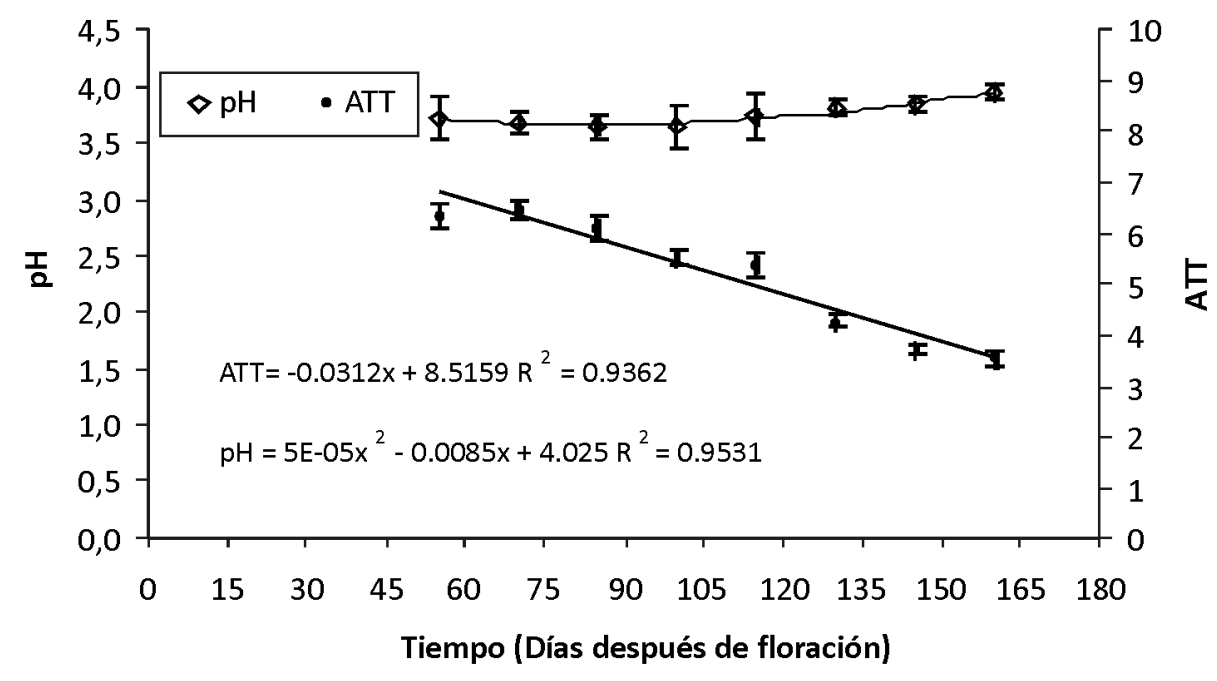

Figura 5. Comportamiento del pH y la ATT en el crecimiento y desarrollo de frutos de champa. Las barras verticales indican la desviación estándar $(n=10)$. 
$0,85^{\circ}$ Brix y, en el momento de la cosecha, presentaron $11,58 \pm 0,62^{\circ}$ Brix; la velocidad de aumento fue de 0,0427º Brix $\cdot$ dia $^{-1}$ (Figura 6).

En la cosecha, los valores de SST fueron mayores que los reportados para C. phae (Vallilo et al. 2005), Eugenia klotzschiana (Vallilo et al. 2003), Myrciaria trunciflora Berg., E. uniflora L., muy similares a los encontrados en Pisidium guajava L. (Lajolo, 2001) y Actinidia deliciosa (Manolopoulou E Papadopoulou, 1998), indicando que la champa es un fruto dulce. El SST está constituido por un 80 a 95\% de azúcares y la SST, se encuentra asociada con los azúcares disueltos en el jugo celular (Osterloh et al. 1996). Estos autores afirman que la cantidad de azúcares depende de múltiples factores: la variedad, el rendimiento asimilatorio de las hojas, la relación hoja/ fruto, las condiciones climáticas, el estado de desarrollo y la madurez.

Fischer E Martínez (1999) mencionan que cuando el fruto de la uchuva presenta en su contenido niveles de azúcares muy altos, éste ha alcanzado su madurez fisiológica, lo que coincide con lo hallado, para la champa, a los 160 DDF. La acumulación de azúcares está asociada con el desarrollo de una adecuada calidad; éstos son incorporados al fruto, principalmente, desde la corriente de fotosintetizados, más que por la degradación de las reservas de almidón del fruto (Heredia et al. 1997). Al respecto, Chitarra E Chitarra (2005) afirman que a medida que los frutos maduran, parte de los sólidos se transforman en azúcares simples, como glucosa, fructosa y sacarosa, generando aumento en los contenidos de sólidos solubles.

En concordancia con lo encontrado para los frutos de champa, se sabe que en guayaba los sólidos solubles se incrementan gradualmente con el desarrollo del fruto (Mercado et al. 1998), lo mismo que se reporta para arazá (Hernández et al. 2007).

A través del crecimiento del fruto de champa, la RM ascendió linealmente hasta la cosecha (Figura 6); a los 160 DDF, la RM fue de 3,28 $\pm 0,23$, momento en el que, posiblemente, se alcanza el punto óptimo de cosecha, porque la concentración de SST es alta, la ATT es mínima y, además, los frutos cambian la coloración de la corteza de verde a amarillo, criterio que, según López E Rodríguez (1995), indica la madurez de consumo.

Pinzón et al. (2007) afirman que en los frutos climatéricos el aumento de la RM posiblemente ocurre cuando alcanzan la tasa respiratoria máxima y desdoblan rápidamente sus reservas (ácidos orgánicos), como respuesta al incremento de su metabolismo y, en consecuencia, la RM se incrementa (Kays, 2004). Osterloh et al. (1996)

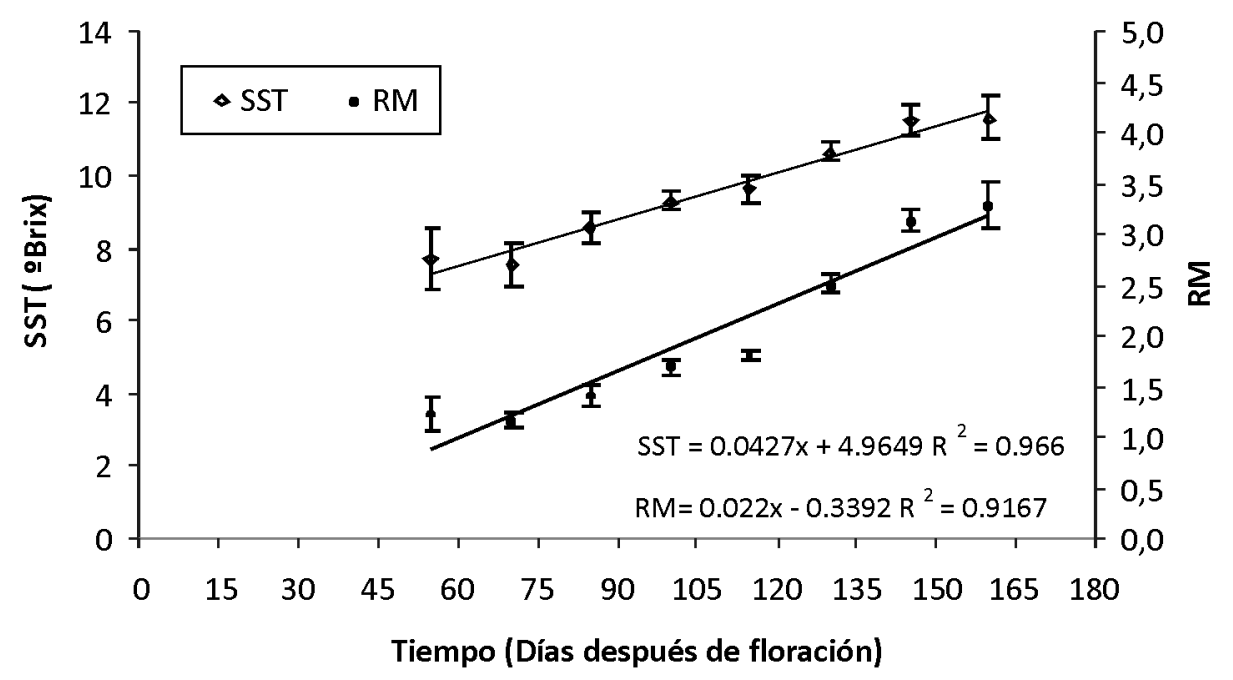

Figura 6. Comportamiento de los SST y la RM en el crecimiento y desarrollo de frutos de champa. Las barras verticales indican la desviación estándar $(n=10)$. 
afirman la importancia de la relación entre SST y ATT en el sabor del fruto y del jugo, teniendo en cuenta que cuando el fruto tiene un contenido alto de azúcares, el nivel de los ácidos debe ser suficientemente elevado para satisfacer el gusto del consumidor.

La RM fue superior a la reportada para C. phae, la cual, presentó una relación de madurez de 0,15 (Vallilo et al. 2005). Sin embargo, la champa muestra valores superiores en SST y en ATT, lo que la convierte en una fruta más dulce y más ácida. Es esta característica la que le da un sabor muy agradable y le genera gran aceptación entre los consumidores.

Se determinó que en el municipio de Miraflores, Boyacá, el crecimiento y el desarrollo del fruto de champa tiene una duración de 160 días después de la floración, momento en el cual fue realizada la cosecha. El crecimiento en masa fresca y en seca siguió un comportamiento sigmoidal simple. El fruto revela alta firmeza en sus estados iníciales, pero a medida que va madurando, se ablanda de forma drástica y presenta baja firmeza en la cosecha, además, es un fruto dulce y ácido a la vez.

AGRADECIMIENTOS: A María Inés López Martín, por permitir la toma de muestras en la finca La Playa y compartir información sobre el manejo del cultivo. A la Dirección de Investigaciones (DIN), de la Universidad Pedagógica y Tecnológica de Colombia, por el apoyo financiero, en el marco del proyecto de capital semilla, Convocatoria 019 de 2008, "Crecimiento, desarrollo y poscosecha del fruto de champa (Campomanesia lineatifolia REP)", SGI 572. Conflictos de intereses: El presente artículo de investigación no presenta ningún conflicto de interés que afecte su publicación, pues todos los autores participaron integralmente en el desarrollo de la investigación y del manuscrito.

\section{BIBLIOGRAFÍA}

1. ANDRADE, J. de S.; ARAGÃO, C.G.; FERREIRA, S.A. do N. 1993. Caracterização física e química dos frutos de Araçá-Pêra (Psidium acutangulum D. C.). Acta Amazônica (Brasil). 23(2):213-217.

2. CHITARRA, M.I.F.; CHITARRA, A.B. 2005. Pós-colheita de frutos e hortaliças: fisiologia e manuseio. Lavras: ESAL-FAEPE, 785p.
3. COOMBE, B.G. 1960. Relationship of growth and development to changes in sugars, auxins, and gibberellins in fruit of seeded and seedless varieties of Vitis vinifera. Plant Physiology (EUA). 35:241-250.

4. DÍAS, D.C.F. 2001. Maturação de sementes. Seed News, Pelotas (Brasil). 5(6):22-25.

5. FISCHER, G.; MARTÍNEZ, O. 1999. Calidad y madurez de la uchuva (Physalis peruviana L.) en relación con la coloración del fruto. Agronomía Colombiana. 16(1-3):35-39.

6. GARCÉS de G, E. 1987. Estudio anatómico y de los procesos de crecimiento del fruto del guayabo (Psidium guajava L). Agronomía Colombiana. 4: 23-30.

7. GEMTCHÜJNICOV, I.D. 1976. Manual de taxonomía vegetal: plantas de interesse econômico, agrícola, ornamentais e medicinais. São Paulo: Ceres, 368p.

8. HEREDIA, J.; SILLER, J.; BÁEZ, M.; ARAIZA, E.; PORTILLO, T.; GARCÍA, R.; MURY, M. 1997. Cambios en la calidad y el contenido de carbohidratos en frutas tropicales y subtropicales a nivel de supermercado. Proc. Interamer. Soc. Trop. Hort. (Brasil). 41:104-109.

9. HERNÁNDEZ, M.S.; MARTÍNEZ, O.; FERNÁNDEZTRUJILLO, J.P. 2007. Behavior of arazá (Eugenia stipitata Mc Vaugh) fruit quality traits during growth, development and ripening. Sci. Hort. (Holanda). 111:220-227.

10. HERNÁNDEZ-GIL, R.; BAUTISTA, D. 1977. Crecimiento y cambios bioquímicos durante el proceso de maduración de la mora (Rubus glaucus BENTH.) (a) Agronomía Tropical. 27(2):225-233.

11. KAYS, S. 2004. Postharvest biology. Ed. Exon Press (Athens, Georgia). 568p.

12. KENDER, W.J.; DESROCHERS, J.C. 1970. Changes in endogenous auxin, gibberellin-like substances, end inhibitors in developing lowbush blueberry fruit. J. Amer. Soc. Hort. Sci. 95(6):699-702. 
13. LAGUADO, N.; PÉREZ, E.; ALVARADO, C.; MARÍN, M. 1999. Características fisicoquímicas y fisiológicas de frutos de guayaba de los tipos Criolla Roja y San Miguel procedentes de dos plantaciones comerciales. Rev. Fac. Agron. (LUZ) (Venezuela). 16:382-397.

14. LAJOLO, M.F. 2001. Tabela brasileira de composição de alimentos. Disponible desde Internet en: www. fcf.usp.tabela. (con acceso 03/12/01).

15. LEGRAND, C.D.; KLEIN, R.M. 1977. Mirtáceas: Campomanesia. In: Reiz, P.R. (ed.) Flora ilustrada catarinense. Itajaí: Herbário Barbosa Rodrigues, p.573-623.

16. LÓPEZ, M.; RODRÍGUEZ, J. 1995. Diagnóstico del mercadeo de la champa en el Municipio de Miraflores, Boyacá. Trabajo de grado para optar al titulo de Tecnólogo en Mercadeo. Instituto de Educación Abierta y a distancia. Universidad Pedagógica y Tecnológica de Colombia. Tunja. 86p.

17. MANOLOPOULOU, H.; PAPADOPOULOU, P. 1998. A study of respiratory and physico-chemical changes of four kiwifruit cultivars during cool-storage. Food Chemistry (Reino Unido). 63(4):529-534.

18. MARSCHNER, H. 2002. Mineral nutrition of higher plants. $2^{\text {nd }}$ ed. Ed. Academics Press (London). 889p.

19. MEDEIROS, C.A.B.; RASEIRA, M.C.B. 1998. A cultura do pessegueiro. Brasilia: Embrapa-SPI, 350p.

20. MERCADO-SILVA, E.; BENITO-BAUTISTA, P.; GARCIA-VELASCO, M. A. 1998. Fruit development, harvest index and ripening changes of guavas produced in central México. Postharvest Biol. Technol. (Holanda). 13:143-150.

21. MINIC, Z.; JOUANIN, L. 2006. Plant glycoside hydrolases involved in cell wall polysaccharide degradation. Plant Physiol. Biochem. 44:435-449.

22. NAKASONE, H.; PAULL, R.E. 1999. Guava. In: Tropical Fruits, CABI Publ. (chapter 7). p.149-172.
23. NANOS, G.D.; KADER, A.A. 1993. Low $\mathrm{O}_{2}$-induced changes in $\mathrm{pH}$ and energy charge in pear fruit tissue. Postharvest Biol. Technol. 3:285-291.

24. ÖPIK, H.; ROLFE, S. 2005. The physiology of flowering plants. 4th ed. Cambridge University Press (Cambridge). 392p.

25. OSTERLOH, A.; EBERT, G.; HELD, W.H.; SCHULZ, H.; URBAN, E. 1996. Lagerung von Obst und Südfrüchten. Verlag Ulmer (Stuttgart). 253p.

26. PINZÓN, I.; FISCHER, G.; CORREDOR, G. 2007 Determinación de los estados de madurez del fruto de la gulupa (Passiflora edulis Sims.). Agronomía Colombiana. 25(1):83-95.

27. REID, M.S. 2002. Maturation and maturity indices. En: Kader, A.A. (ed.) Postharvest technology of horticultural crops. $3^{\text {th }}$ ed. U. California, Agricultural and Natural Resources, Publication 3311 (Oakland). p.55-62.

28. RODRÍGUEZ, M.; ARJONA, H.E.; CAMPOS. H.A. 2006. Caracterización fisicoquímica del crecimiento y desarrollo de los frutos de feijoa (Acca sellowiana Berg) en los clones 41 (Quimba) y 8-4. Agronomía Colombiana. 24(1):54-61.

29. SALISBURY, F.; ROSS, C. 1994. Fisiología vegetal. Grupo Editorial Iberoamérica, (México). p.309318.

30. VALLILO, M.I.; BAITELLO, J.B.; LAMARDO, L.; LOBANCO, C.M. 2003. Composição química do fruto de Eugenia klotzschiana Berg. (MYRTACEAE). Ver. do Instituto Florestal (Brasil). 15(1):33-44.

31. VALLILO, M.I.; GARBELOTTI, M.L.; DE OLIVEIRA, E.; LAMARDO, L. 2005. Características físicas e químicas dos frutos do cambucizeiro (Campomanesia phaea). Rev. Bras. Frutic. 27(2):241-244.

32. VILLACHICA, H. 1996. Frutales y Hortalizas promisorios del Amazonas. Tratado de Cooperación Amazónica, Secretaría Pro Tempore (Lima). p.181-185. 
33. VILLAMIZAR, F. 2001. Manejo tecnológico postcosecha de frutas y hortalizas. Aspectos Teóricos. Ed. Unidad de Publicaciones. Facultad de Ingeniería, Universidad Nacional de Colombia (Bogotá). 149p.

34. VICKERY, H.B. 1952. The behavior of isocitric acid in excised leaves of Bryophyllum calicinum during culture in alternating light and darkness. Plant Physiol. 27:9-17.
35. ZARRA, I.; REVILLA, G. 1993. Pared celular. Estructura y función. En: Azcon-Bieto, J.; Talón, M. (eds.) Fisiología y bioquímica vegetal, McGraw-Hill Interamericana de España (Madrid). p.1-24.

Recibido: Abril 11 de 2009

Aceptado: Septiembre 5 de 2009 